\title{
ANALYSIS OF TEMPORAL FACTORS INFLUENCING MINIMUM DWELL TIME DISTRIBUTIONS
}

\author{
TIMOTHY PEDERSEN ${ }^{1}$, THOMAS NYGREEN ${ }^{2} \&$ ANDERS LINDFELDT $^{1}$ \\ ${ }^{1}$ Sweco, Norway \\ ${ }^{2}$ The Norwegian Railway Directorate, Norway
}

\begin{abstract}
The minimum dwell time is an important part of railway timetable planning. Due to its stochastic behaviour, the minimum dwell time should be considered to create resilient timetables. While there to our knowledge has been significant focus on how to determine and estimate dwell times, little research has been carried out regarding temporal and running direction variations of these. In this paper we examine how the minimum dwell time varies depending on temporal factors such as the time of the day, day of the week and time of the year. We also examine how it is affected by running direction and station type. The minimum dwell time is estimated by means of track occupation data. A method is proposed to ensure that only minimum dwell times and not planned dwell times are acquired from the track occupation data. The results show that on an aggregated level, the average minimum dwell times in both running directions at a station are similar. However, when temporal factors are considered, there are significant variations. The minimum dwell time varies throughout the day with peak hours having the longest dwell times. It is also found that the minimum dwell times are influenced by weekday, and in particular weekends are found to have lower minimum dwell times than most other days. The findings show that there is a potential to significantly improve timetable planning by taking minimum dwell time variations into account.
\end{abstract}

Keywords: minimum dwell time, operations quality, timetable planning, track occupation data.

\section{INTRODUCTION}

The minimum dwell time is a determinant factor for railway timetables when planning for efficient operation. An insufficiently scheduled dwell time can cause delays to the current and subsequent trains because of strict time schedules (secondary delays). An excessively large scheduled dwell time can however lead to inefficient train operation [1], [2]. Estimating these parameters appropriately can make timetables more robust, increase punctuality and thus enhance the quality of railway as a transport mode.

While previous research has focused a lot on headway and running time for timetable planning, there has been little focus on minimum dwell times and their temporal variations. Earlier research has focused on creating models for dwell times, with parameters such as passenger volumes, door width, location of platform access, train service type and which hour and day it is.

Riksrevisionsverket, which is a Swedish authority, found that dwell times at stations were based on the needed time in off-peak hours, not in peak hours. During the peak hours, the dwell times were basically sufficient, but there was no slack during these periods. This made the train schedule vulnerable to disturbances [3]. Buchmüller et al. divided the dwell time into five sub-processes which were: door-unblocking, the time it takes for the doors to open, boarding and alighting of passengers, door closing and train dispatching. The dwell times were considered as random processes that depended on several other factors, such as the number of passengers at a station and how passengers were distributed throughout the station area [4]. Wiggenraad found that the dwell time depended on type of train service. In The Netherlands, local trains had dwell times of 45-60 seconds on the average. He also found that the dwell times were about the same during peak and off-peak hours with some few 
exceptions. The data collection consisted of having people on the stations that measured the dwell times using stopwatches [5]. However, it is unknown if the dwell times included scheduled dwells at the stations.

In contrast to what Wiggenraad found, Li et al. found that in The Netherlands dwell times varied significantly during the day at short stop stations, at which trains depart as soon as the alighting and boarding process has finished. He also found that dwell times during the working days were similar, while Saturdays and Sundays were different from any other day. Li et al. used track occupation data from the Dutch train describer system (TNV). He estimated the dwell times at different hours and days and the results showed that working days had significantly larger dwell times compared to the weekend. Also, he found that the dwell times varied significantly with time of day, with peak-hours having the longest dwell times [6].

However, it is not known if such dwell time variations apply for stations in other countries, as travel patterns can be different. Stations with other characteristics than short stop stations, such as train stations at public transport hubs, have also not been examined for such variations. Those two types of stations can have very different passenger volumes, which can affect the dwell time variations differently.

In this paper we address the following research questions:

- What are the statistics of dwell times at two stations with different characteristics?

- How does hour, weekday, as well as running direction, affect the dwell times at those stations?

The paper is organised as follows: Section 2 describes how the examined stations have been chosen, Section 3 describes the method for data acquisition. The results are presented in Section 4. Finally, the conclusion is presented at the end of this paper.

\section{SELECTION OF DATA SOURCE AND STATIONS}

In this paper we are using arrival and departure times from the Norwegian traffic information and monitoring system TIOS. This enables the acquisition of a large number of observations at each station, as the system records all trains running on the Norwegian network.

Two stations were chosen on the following criteria:

- The first station had to be an intermediate local train station on a double track line

- The second station had to be a large station at a public transport hub

- The examined stations and tracks had to have as much similar train types as possible, so that train dynamics did not differ too much

- No single-track lines because of possibly secondary delays

Billingstad (BST) and Lysaker (LYS) stations in respectively Asker and Bærum municipality, west of Oslo, have been chosen based on these criteria. While BST is a local train station on the double track Drammen line (Norwegian: Drammenbanen), also used by freight trains, LYS station was in 2006 one of Norway's biggest stations measured in terms of passengers [7]. The station serves both Drammen line and Asker line (also a double track line) with four platform tracks. East of the station, two double track lines (Askerbanen and Drammenbanen) merge into one double track line towards the city centre of Oslo. Eastbound traffic from different lines therefore need to be synchronised at Lysaker before departing towards to Oslo. For eastbound trains, dwell times at Lysaker will therefore include some secondary delays. Even though there are methods for reducing the effect of secondary delays 
when estimating dwell times [2], they have not been implemented in this paper and are thus a possibility for future improvement of the proposed method. Removing secondary delays will also make it possible to estimate dwell times on single track lines.

Track 1 and 4 at this station, respectively for westbound and eastbound trains, serve the local trains on Drammenbanen, whereas Tracks 2 and 3 are used for regional/IC trains on Askerbanen. Due to problems with calibrating the departure times of the FLIRT trainsets heavily used on Askerbanen, which we will touch upon in the next section, only data from trains on Drammenbanen have been used. This means the same trains are analysed at Lysaker as at Billingstad, avoiding differences caused by differing rolling stock.

To ensure enough data to perform the analysis, we analysed data from the period $15 / 09 / 2015-15 / 03 / 2016$, which is equal to 183 days.

\section{METHODOLOGY}

The dwell time can simply be defined as the difference between the time of departure and time of arrival at a station. However, the arrival and departure times in TIOS are not measured when the trains actually stop and start at the platform. Like the data from the Dutch TNV system used by Li et al. in [6], our data from TIOS are exact times for when the trains entered certain track sections before arrival and after departure, as seen in Fig. 1.

Network-wide data tools like TNV in the Netherlands, TIOS in Norway and TRUST in the UK are relied upon for calculating performance indicators and enforcing performance regimes. The extensive set of recorded arrivals and departures is also an essential input when performing traffic analyses or calibrating running time calculators used in traffic simulations and timetable planning. However, without proper calibration, the use of the raw data introduces systematic errors in the statistics and analyses. As the registered arrival is always before the actual arrival, and the registered departure always later than the actual departure, registered dwell times become longer than the actual dwell times. Likewise, the registered running times between stations are shorter than the actual running times.

By assuming certain deceleration rates, $\mathrm{Li}$ et al. used the registered entry speed into the block section prior to the station to estimate the time it would take for the train to stop at a fixed point at the station. Consequently, by adding the deceleration time to the block occupation time prior to the station, the estimated actual time of arrival was obtained. Likewise, for the time of departure from the station, they assumed acceleration rates based on the rolling stock type and train length. Thus, they could estimate how much time it would take to drive from the stopping point at the station to the next block section, from which time event data was obtained. By subtracting the estimated acceleration time from the registered

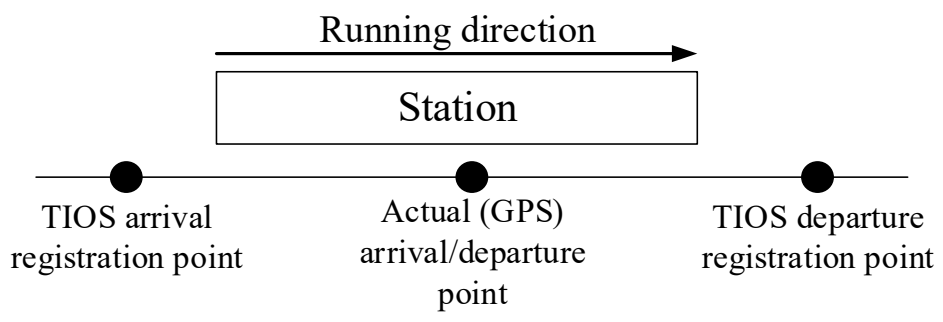

Figure 1: TIOS arrival and departure registration points. 
block occupation time after the station, the estimated actual departure time was acquired. The estimated dwell time was thus the estimated departure time minus the estimated arrival time In the UK, Network Rail maintains a list of berth offsets that are automatically added to or subtracted from the time a train occupies a track circuit to obtain the official arrival and departure times stored in their TRUST system. The berth offsets are obtained through field observations and are regularly revised in a process of offset reviews.

The interface supplying track occupation times to TIOS also supports berth offsets, but it is currently only used in extreme cases, meaning that the data is mostly uncalibrated. In 2013, the Norwegian National Railway Administration (later split into Bane NOR and the Norwegian Railway Directorate) initiated a project to calibrate the TIOS data using arrival and departure times recorded by GPS equipment already installed in some trains operated by NSB as part of an automated passenger counting (APC) system. While the APCs did record the actual arrival and departure at the platform, and therefore has a better accuracy than TIOS, these time measurements are less precise than those measured by the track circuit equipment, and only covered less than one in five trains. For each combination of station, track and direction a robust mean offset was calculated, producing a list of coefficients that could be used to calibrate the entire TIOS dataset.

In 2016 The Norwegian National Railway Administration and Sweco repeated the project, assuring the quality of the method devised in 2013. This time, we used two years of GPS recorded times, also exploiting that a larger share of the train fleet had been equipped with APCs. It is this data set that is used in this study.

Neither of our selected stations are short stop stations, as trains in Norway have to wait for the scheduled departure time at all stations, with the exception of halts on the line between stations and conditional stops. In our analysis, we have therefore only examined delayed trains, which are trains that arrive after the scheduled departure time at a station. Our assumption is that delayed trains, will depart as soon as possible after the boarding and alighting process has finished. Thus, this criterion is crucial when determining the minimum dwell time, and not the nominal dwell time. The latter can possibly include scheduled dwell times and increased dwell due to early-arriving trains, which is not in our interest.

\section{RESULTS}

\subsection{Dwell time statistics}

Table 1 shows the results from the dwell time calculations for the two stations. For each station, the average dwell time for western and eastern direction is not found to be significantly different. However, the same thing cannot be said when the two stations are compared to each other, as they show significantly different dwell times. LYS station has an average dwell time that is around 7 seconds longer than BST station and a standard deviation that is approximately twice as large. This difference can be caused by different passenger volumes, as LYS station is a public transport hub with more passengers than BST.

The results also show that the number of observations in western direction is bigger than in eastern direction, see Table 1 . To see if this bias was caused by looking only at delayed trains, we looked at the number of observations for all registered trains, see Table 2. Our analysis shows that the numbers of observations in both directions for all trains are very similar. Thus, there is a direction bias when we only look at delayed trains. 
Table 1: Analysis of minimum dwell times of delayed trains. Data from 15/9-2015 to 15/3-2016.

\begin{tabular}{|l|l|l|}
\hline Parameter & \multicolumn{2}{|l|}{ Station } \\
\hline & BST & LYS \\
\hline Direction west & 1078 & 1520 \\
\hline Number of observations & $31.88 \mathrm{sec}$ & $38.50 \mathrm{sec}$ \\
\hline Mean DT & $7.78 \mathrm{sec}$ & $14.10 \mathrm{sec}$ \\
\hline Standard deviation DT & \multicolumn{3}{|l|}{} \\
\hline Direction east & 682 & 657 \\
\hline Number of observations & $32.67 \mathrm{sec}$ & $39.46 \mathrm{sec}$ \\
\hline Mean DT & $9.17 \mathrm{sec}$ & $4.67 \mathrm{sec}$ \\
\hline Standard deviation DT
\end{tabular}

Table 2: Analysis of minimum dwell times. Data from 15/9-2015 to 15/3-2016.

\begin{tabular}{|c|c|c|}
\hline \multirow[t]{2}{*}{ Parameter } & \multicolumn{2}{|l|}{ Station } \\
\hline & BST & LYS \\
\hline \multicolumn{3}{|l|}{ Direction west } \\
\hline Number of observations & 2498 & 3151 \\
\hline Mean DT & $33.71 \mathrm{sec}$ & $42.33 \mathrm{sec}$ \\
\hline Standard deviation DT & $7.95 \mathrm{sec}$ & $15.10 \mathrm{sec}$ \\
\hline \multicolumn{3}{|l|}{ Direction east } \\
\hline Number of observations & 2581 & 3090 \\
\hline Mean DT & $34.43 \mathrm{sec}$ & $53.70 \mathrm{sec}$ \\
\hline Standard deviation DT & $8.64 \mathrm{sec}$ & $25.58 \mathrm{sec}$ \\
\hline
\end{tabular}

\subsection{Dwell times and different hours on working days}

The analysed dwell times have been classified after their estimated time of arrival at a station and are presented by using box plots. This way their distributions are visualised in a systematic manner. Since the data in the boxplots are based on the arrival hour, an estimated time of arrival of a train at 08:59:59 counts as an arrival at 8 o'clock. The dwell times are furthermore separated into western and eastern direction to examine possible differences.

The dwell times at station BST in direction west are shown in Fig. 2 and it can be noticed that the peak hours influence the dwell time: After the morning peak hours (7-9), the dwell times become smaller until around 11 o'clock, from which they increase. At 16 o'clock, during the afternoon peak hours, the dwell times peak before it decreases yet again. T-tests show that peak hours are significantly different from off-peak hours, except for the morning peak hour at 9 o'clock. Since this station lays west of Oslo, the rush direction in the morning will be in eastern direction, and vice versa in the afternoon. The observed afternoon peak at 16 o'clock in western direction can therefore be expected, since large amounts of commuters arrive home. In the morning hours there is no prominent peak, probably due to few passengers going in the non-rush direction.

The results for the dwell times in eastern direction, shown in Fig. 3, show some similar 
tendencies as in the other direction: After the morning peak hours, the dwell times seem to decrease, but they do not have a prominent peak in the afternoon peak hours as it had in western direction. This is reasonable as the morning hours will be in the rush direction, whereas the afternoon hours will not be that. Significance testing shows that the afternoon peak hours are not different from the off-peak hours.

The dwell times at station LYS in western direction are shown in Fig. 4 and show that the peak hours seem to have larger dwell times than the off-peak hours. This is also the case in eastern direction, shown in Fig. 5, but the data variance in the peak hours seem to be larger than in western direction. Our analysis shows that the peak hours have significantly longer minimum dwell times than off-peak hours.

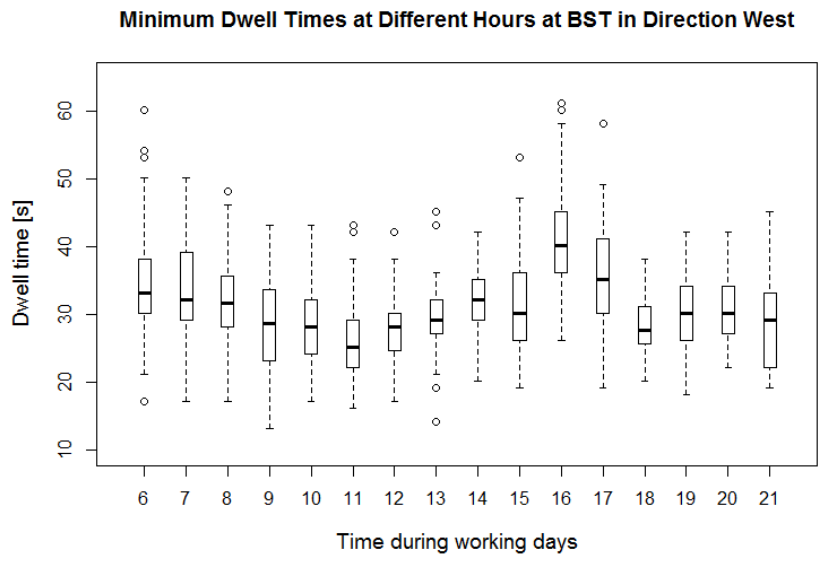

Figure 2: Box plot of minimum dwell times at different hours at BST station in direction west.

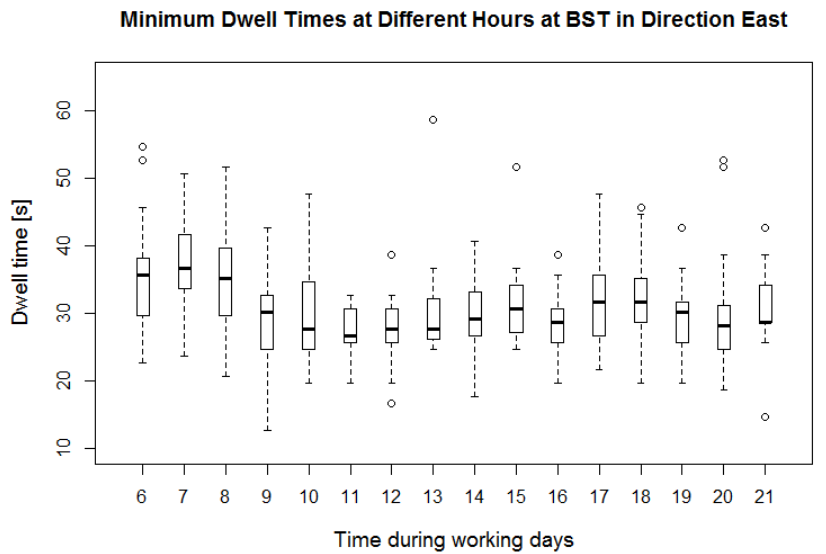

Figure 3: Box plot of minimum dwell times at different hours at BST station in direction east. 


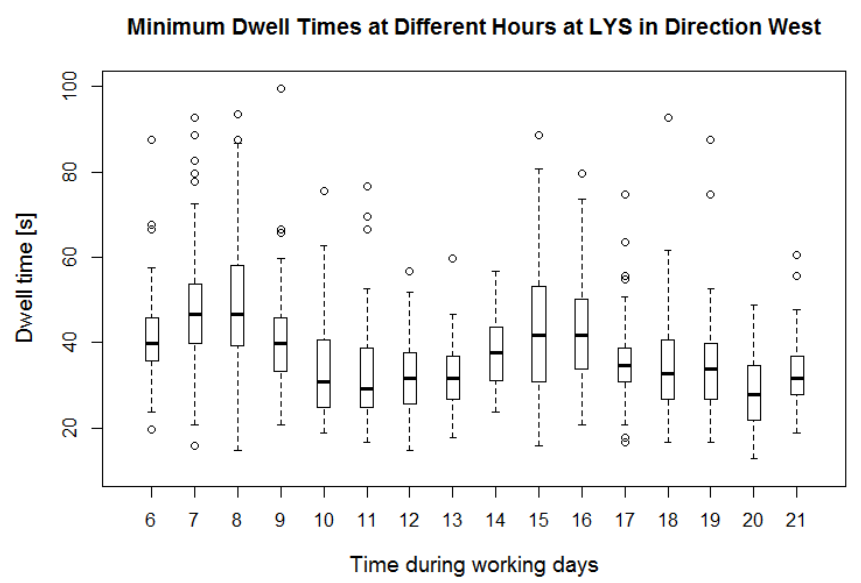

Figure 4: Box plot of minimum dwell times at different hours at LYS station in direction west.

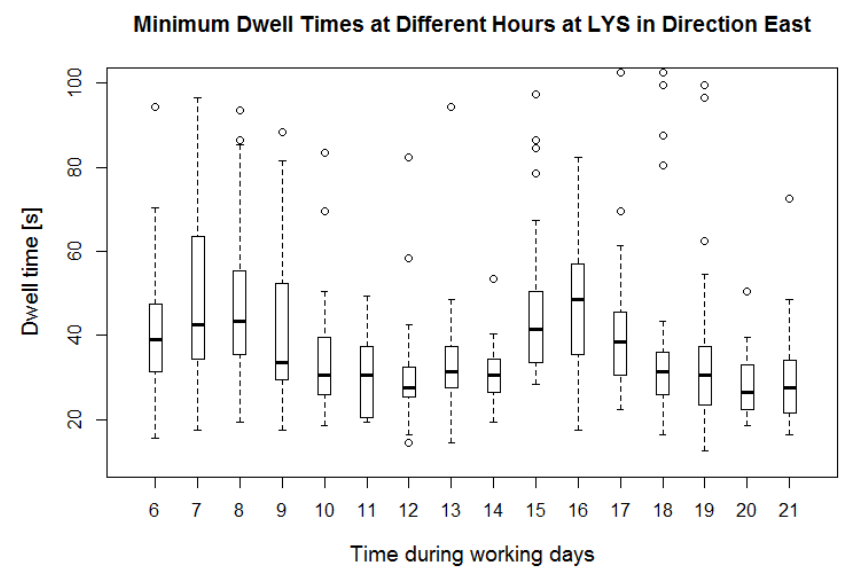

Figure 5: Box plot of minimum dwell times at different hours at LYS station in direction east.

The hourly variation can also be used in timetable planning, e.g. by planning with a larger dwell time during peak hours. This can be useful for trains that only operate in peak hours. However, if increased dwell times are added to full-day service trains, it should also be added to off-peak hours to keep a cyclic timetable. Eventually, this can lead to a more inefficient train operation. Dwell times that are only altered in peak hours for some full-service trains, would lead to different average velocities. This can cause problems in terms of capacity as headways become uneven. A solution to this could be have larger dwell times only for a few stations with high passenger volumes, so that the headway difference stays at an acceptable level. 
4.3 Dwell times and different days of the week

The dwell times have in this section been categorised according to the day of the estimated time of arrival. Fig. 6 shows the dwell times at BST station in direction west, and the boxplots for Monday to Saturday show that the medians are visibly not very different, except for Sunday's median that is visibly lower. Significance testing of pairs of days shows that Sunday is different from any other days. In eastern direction, Fig. 7, significance testing shows that only Monday is different from Saturday and Sunday. The rest of the days show no significant difference.

In Fig. 8 and Fig. 9, the dwell times at LYS are shown. Both Saturday and Sunday are significantly different from almost any other day in the week, but the dwell times for Saturday and Sunday are found to be similar.

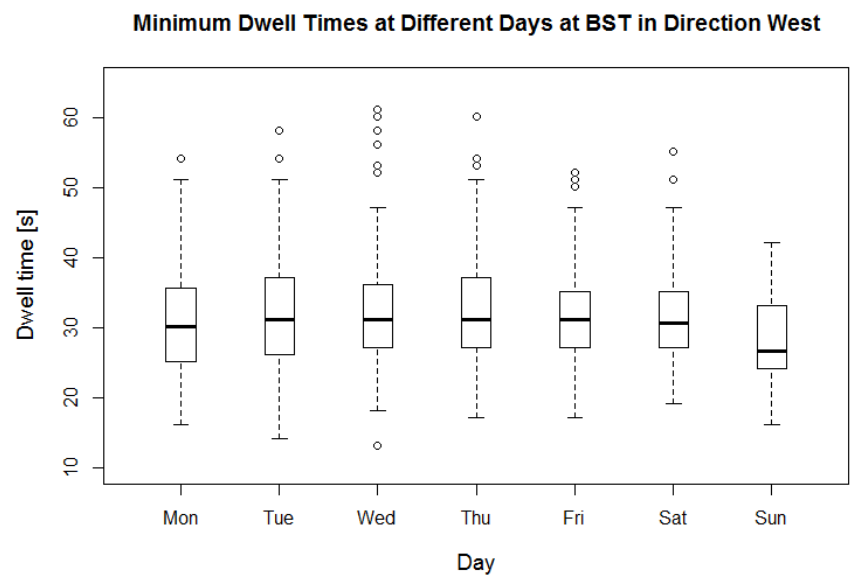

Figure 6: Box plot of minimum dwell times at different days at BST station direction west.

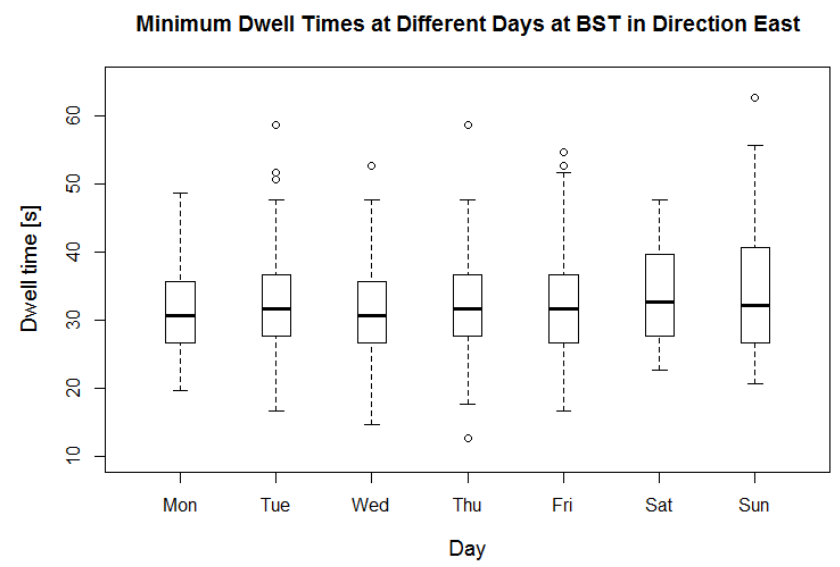

Figure 7: Box plot of minimum dwell times at different days at BST station direction east. 


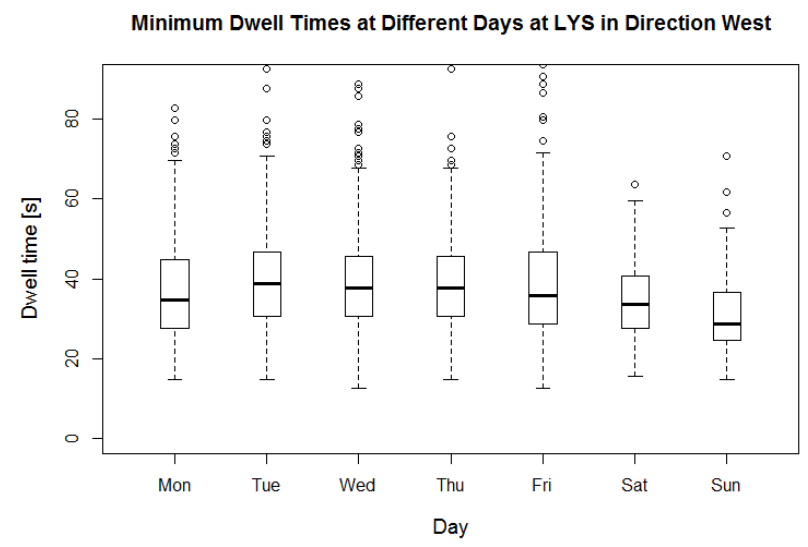

Figure 8: Box plot of minimum dwell times at different days at LYS station direction west.

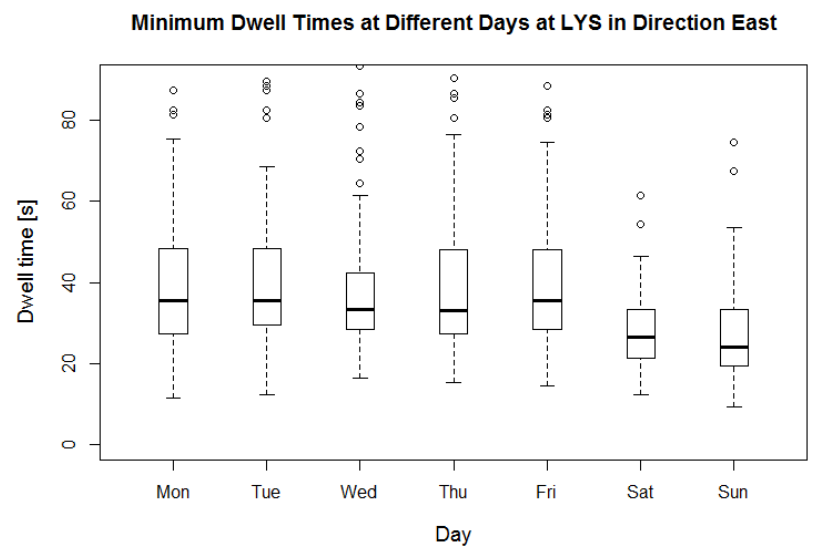

Figure 9: Box plot of minimum dwell times at different days at LYS station direction east.

The differences between the two stations can indicate that the daily passenger volumes are more constant at the local train station BST throughout the week, whereas the dwell times during working days at LYS station are influenced by large volumes of commuters. One solution to this could be to increase the number of trains in the rush hours, but this will not necessarily be a satisfactory solution as delays are large and only a limited number of trains can be sent through a station during a time period. A better solution in order to cope with the increased dwell times, can be to increase the number of wagons for each train. However, success with this solution will depend partly on station design: Will the passengers be distributed evenly throughout the platform, so that each wagon has an equal number of boarding passengers? If the passengers on the platform instead are highly concentrated in the middle of the train, which can happen with some station designs, more wagons are of little help. 


\subsection{Dwell times and different weeks}

The dwell times from 24 successive weeks have been analysed and plotted in Fig. 10 to Fig. 13. It can be observed that the dwell times are inconsistent throughout the period, with some weeks having large variations, whereas others have very little variance. The latter is in some cases due to few observations. The results can also indicate that travel patterns change depending on which time of the year it is. In Fig. 12 there is however a lowering of the dwell times from week 51. Although the causes of this are unknown, it is thought that changes to the platform or infrastructure can have contributed to this.

In future research, weekly dwell time variations should be examined closer to try and isolate factors that influences this. This could lead to a greater understanding about this variation.

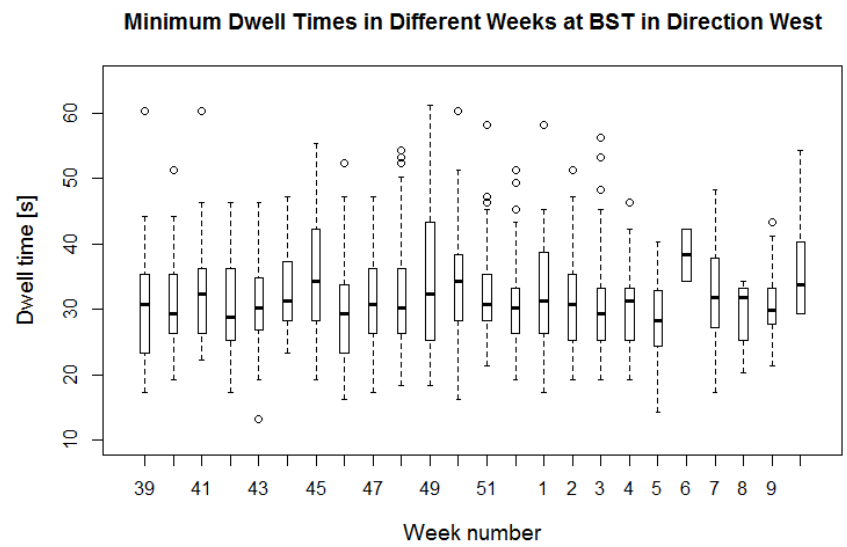

Figure 10: Box plot of minimum dwell times in different weeks at BST station in direction west.

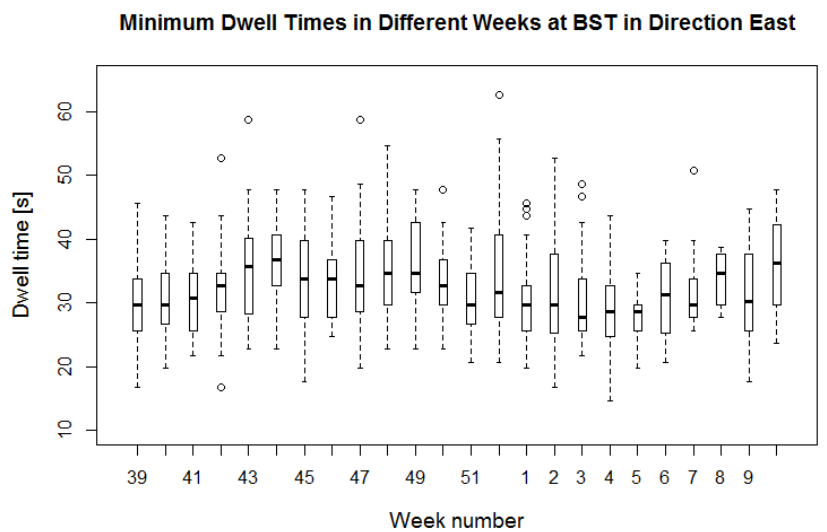

Figure 11: Box plot of minimum dwell times in different weeks at BST station in direction east. 


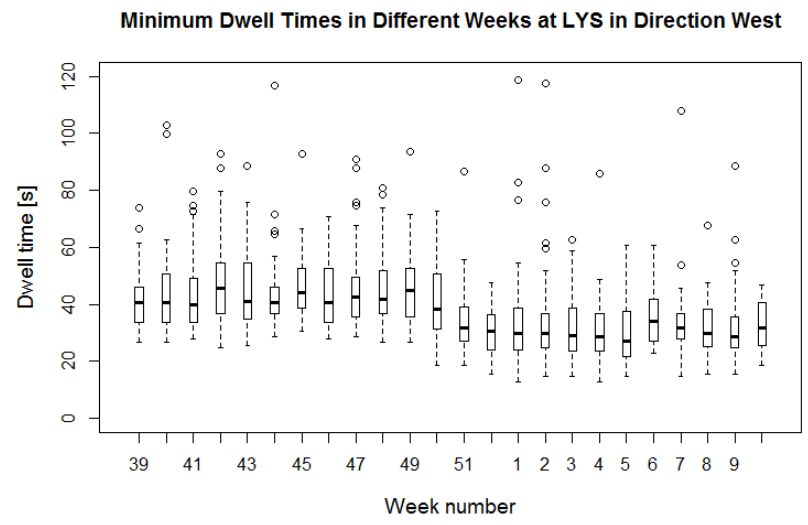

Figure 12: Box plot of minimum dwell times in different weeks at LYS station in direction west.

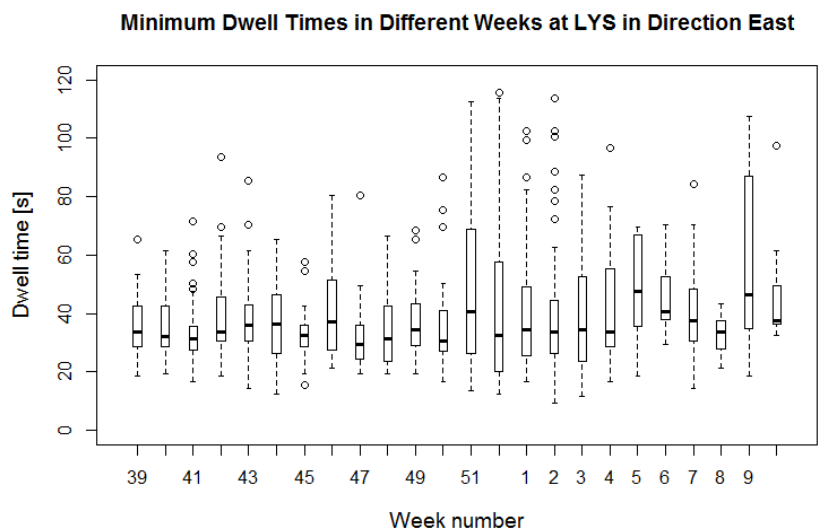

Figure 13: Box plot of minimum dwell times in different weeks at LYS station in direction east.

\section{CONCLUSION}

In this paper minimum dwell times at two different types of stations have be examined with regards to factors as running direction, hour, weekday and week. The analysed data come from track occupation data which have been calibrated to estimate the actual arrival and departure times. The results show that the dwell times have significant variations when the examined parameters are considered. This suggests that both time of day and the direction of the trains must be included in the analysis, or problems with long dwell times might be underestimated. The reason is that the longest dwell times in many cases occur during rush hour and in the direction of rush traffic, i.e. long dwell time coincides with times and areas of high capacity utilisation. This lead to a proposal to utilise longer trains instead of more trains during the rush hours. 


\section{ACKNOWLEDGEMENTS}

This research was financially supported by Sweco Norway. Very special thanks go to The Norwegian Railway Directorate (NRD) for providing us data, as well as a general contribution to this article. We also thank Thomas Nygreen, Railway Capacity Engineer at $\mathrm{NRD}$, for a thorough and accurate description of the methodology, as well as a general contribution to this article (thomas.nygreen@jernbanedirektoratet.no). We would also like to show gratitude to Erik Natvig, former employee at NRD, for giving us ideas and insights about this topic. Without them, it would have been challenging to complete this article.

\section{REFERENCES}

[1] Jong, J.-C. \& Chang, E.-F., Investigation and Estimation of Train Dwell Time for Timetable Planning, 9th World Congress on Railway Research (WCRR), 2011.

[2] Lindfeldt, A. \& Sipilä, H., Användning av tågpassagedata för estimering av primära störningsfördelningar, Kungliga Tekniska högskolan Transportplanering, ekonomi och teknik (TET) 10044, Stockholm, 2016.

[3] Riksrevisjonsverket, Tågtrafikens punktlighet. Revisjonsrapport. Dnr. 1986: 1091. Riksrevisjonsverket, Stockholm, Sweden, 1986.

[4] Buchmüller, S., Weidmann, U. \& Nash, A., Development of a dwell time calculation model for timetable planning, Institute for Transport Planning and Systems, ETH Zürich. Computers in railways, XI(525), 2008.

[5] Wiggenraad, P., Alighting and Boarding Times of Passengers at Dutch Railway Stations, TRAIL Research School, Delft University of Technology, 2001.

[6] Li, D., Goverde, R.M.P., Daamen, W. and He, H. (2014): Train Dwell Time Distribution at Short Stop Stations. 2014 IEEE 17th International Conference on Intelligent Transportation Systems (ITSC).

[7] Ny Lysaker stasjon. Fra to til fire spor. Nytt dobbeltspor Skøyen - Asker. http://www.banenor.no/contentassets/f27afa75782b493e8a357c7cc8b50e9b/brosjyre_1 ysaker-s_1620924a.pdf. Accessed on: 4 Apr. 2018. 